\title{
Lidil
}

Revue de linguistique et de didactique des langues

$57 \mid 2018$

Démarches créatives, détours artistiques et appropriation des langues

\section{Carole Glorieux, Mémoires professionnels, mémoires d'application et autres travaux de fin d'études (TFE) : concepts utiles et dispositifs didactiques}

Presses universitaires de Namur, collection « Tactiques », $\mathrm{n}^{\circ}$ 10, 2016, $244 \mathrm{p}$.

\section{Carmenne Kalyaniwala}

\section{OpenEdition}

Journals

Édition électronique

URL : http://journals.openedition.org/lidil/4850

DOI : 10.4000/lidil.4850

ISSN : $1960-6052$

Éditeur

UGA Éditions/Université Grenoble Alpes

Édition imprimée

ISBN : 978-2-37747-048-8

ISSN : $1146-6480$

\section{Référence électronique}

Carmenne Kalyaniwala, «Carole Glorieux, Mémoires professionnels, mémoires d'application et autres travaux de fin d'études (TFE) : concepts utiles et dispositifs didactiques », Lidil [En ligne], 57 | 2018, mis en ligne le 01 mai 2018, consulté le 23 septembre 2020. URL : http://journals.openedition.org/lidil/4850 ; DOI : https://doi.org/10.4000/lidil.4850

Ce document a été généré automatiquement le 23 septembre 2020.

(C) Lidil 


\section{Carole Glorieux, Mémoires} professionnels, mémoires d'application et autres travaux de fin d'études (TFE) : concepts utiles et dispositifs didactiques

Presses universitaires de Namur, collection «Tactiques », $n^{\circ} 10,2016$, 244 p.

\section{Carmenne Kalyaniwala}

\section{RÉFÉRENCE}

Carole Glorieux, Mémoires professionnels, mémoires d'application et autres travaux de fin d'études (TFE) : concepts utiles et dispositifs didactiques, Presses universitaires de Namur, collection « Tactiques », $\mathrm{n}^{\circ}$ 10, 2016, $244 \mathrm{p}$.

1 Cet ouvrage est le résultat d'une réflexion élargie qui fait suite à la soutenance d'une thèse doctorale en 2015, sous la direction de Marie-Christine Pollet à l'Université libre de Bruxelles. Il s'inscrit dans le champ de la didactique et se trouve plus particulièrement à la confluence des recherches en littératies professionnelles et universitaires. Le livre est divisé en sept chapitres préfacés par la directrice de thèse de l'auteure. Un préambule identifie les lecteurs destinataires du livre: chercheur-e-s, didacticien-ne-s et enseignant-e-s du supérieur qui encadrent des mémoires à vocation professionnelle.

2 Un chapitre de l'ouvrage est dédié au contexte dans lequel s'inscrit l'auteure - à la jonction entre les littératies professionnelles et les littératies universitaires. Par littératies - avec trois « $t$ »-, l'auteure désigne «l'appropriation des pratiques de lecture et d'écriture dans un contexte donné où, plus globalement, comme le rapport à l'écrit(ure) de l'individu, tout au long de la vie, de la plus petite enfance au milieu professionnel» (p. 28). Le qualificatif « universitaire» vient compléter ce premier 
concept afin de situer l'étude dans une catégorie pertinente des littératies. De plus, suivant la tradition anglo-saxonne, l'auteure préfère la version plurielle du concept, à savoir « littératies universitaires » pour prendre en compte l'« ensemble des pratiques sociales et culturelles de la lecture et de l'écriture dans des contextes particuliers » (Léa, 2008, cité par Glorieux, 2016, p. 31). Le champ d'étude de l'auteure emprunte ses réflexions à trois disciplines: l'analyse du discours, la didactique du français langue maternelle et la pédagogie universitaire.

3 Nous trouvons que le point fort de ce chapitre et du suivant se trouve dans la typologie des genres de discours universitaires (DU) proposée par l'auteure où elle établit une première distinction entre les DU institutionnels, les DU scientifiques et les DU professionnalisés. En se focalisant sur le troisième type de discours, Glorieux se pose des questions sur la place du discours «des professions» (p. 56) au sein des littératies universitaires et professionnelles. L'auteure reprend plus loin la typologie exposée afin de détailler les différents types de mémoires professionnels qui existent sur le terrain.

4 Un autre chapitre est consacré à l'explicitation des concepts et outils théoriques: professionnalisation, réflexivité, scientificité, contrainte, représentation, problématique et problématisation, désignation et polyphonie. Elle propose un « parcours lecturo-scriptural » (expliqué à la p. 100) aux enseignants qui encadrent les travaux professionnalisants.

5 Un nouveau concept-outil conçu par Glorieux est enfin ajouté. L'auteure définit son concept de "collision générique " comme «le choc créé par le fait que des genres de discours différents sont traités ou utilisés comme s'ils appartenaient au même genre, pour former un tout cohérent. Ou plutôt il s'agit selon moi, du lieu textuel de rencontre de genres de discours différents, traités ou utilisés comme s'ils appartenaient au même genre, et formant un nouveau genre, cohérent » (p. 129, italique dans le texte original). Il a le mérite de prendre en considération les difficultés rencontrées par les étudiants-scripteurs du mémoire professionnel. Elle suggère ainsi qu'une meilleure gestion de la collision générique permettrait éventuellement aux étudiants d'un master d'accomplir la tâche de rédiger leurs mémoires professionnels. Une gestion inadaptée de la collision générique se retrouve comme une difficulté principale des étudiants. Plus loin, l'auteure expose une typologie des différentes difficultés rencontrées.

6 Enfin, l'ouvrage propose huit pistes didactiques pour former un-e "mémorant-e » (celui ou celle qui rédige un mémoire) à l'écriture d'un mémoire professionnel. Ces pistes seront utiles pour tout-e enseignant-e souhaitant proposer des dispositifs méthodologiques pour former un étudiant à cette tâche.

7 À la suite de notre lecture, nous pensons que le présent ouvrage peut être salué par les chercheur-e-s didacticien-ne-s qui s'intéressent aussi bien à une réflexion épistémologique qu'à l'application de la théorie au profit d'un terrain professionnalisant. L'auteure nous amène progressivement vers l'outil didactique qu'elle propose dans son ultime chapitre en montrant une connaissance fine du terrain dans les chapitres précédents. Il est ainsi facile de suivre son parcours réflexif.

Que ce soit sur le plan réflexif ou sur le plan praxéologique, l'ouvrage peut donc être d'une grande utilité autant pour les éducateurs-trices (chercheur-e-s, didacticien-ne-s, encadrant-e-s) que pour les étudiant-e-s qui préparent un mémoire professionnel. Le chapitre sept pourrait, à titre d'exemple, être étudié comme un guide d'autoformation 
si l'on essaie d'adapter les activités proposées par l'auteure à des contextes d'étude différents.

\section{AUTEURS}

\section{CARMENNE KALYANIWALA}

LIDILEM, Université Grenoble Alpes \& Université de Lorraine 